\title{
openheart Type 2 myocardial infarction and myocardial injury: eligibility for novel medical therapy to derisk clinical trials
}

\author{
Robert Sykes (D) , ${ }^{1,2}$ Michael Briscoe, ${ }^{3}$ Thomas Krysztofiak, ${ }^{3}$ Oliver Peck, ${ }^{2,3}$ \\ Kenneth Mangion, ${ }^{1,3}$ Colin Berry ${ }^{1,2,3}$
}

\begin{abstract}
- Additional supplemental material is published online only. To view, please visit the journal online (http://dx.doi.org/10.
\end{abstract} 1136/openhrt-2021-001633).

To cite: Sykes R, Briscoe M, Krysztofiak T, et al. Type 2 myocardial infarction and myocardial injury: eligibility for novel medical therapy to derisk clinical trials. Open Heart 2021;8:e001633. doi:10.1136/ openhrt-2021-001633

RS and MB contributed equally.

Received 25 February 2021 Accepted 16 May 2021

A) Check for updates

(c) Author(s) (or their employer(s)) 2021. Re-use permitted under CC BY-NC. No commercial re-use. See rights and permissions. Published by BMJ.

${ }^{1}$ British Heart Foundation Glasgow Cardiovascular Research Centre, Institute of Cardiovascular and Medical Sciences, University of Glasgow, Glasgow, UK

${ }^{2}$ West of Scotland Regional Heart and Lung Centre, Golden Jubilee National Hospital, Clydebank, UK

${ }^{3}$ Department of Cardiology, Queen Elizabeth University Hospital, Glasgow, UK

Correspondence to Professor Colin Berry; colin. berry@glasgow.ac.uk

\section{ABSTRACT}

Background Patients with type 2 myocardial infarction (T2MI) and other mechanisms of nonthrombotic myocardial injury have an unmet therapeutic need. Eligibility for novel medical therapy is generally uncertain. Methods We predefined colchicine, eplerenone and ticagrelor as candidates for repurposing towards novel therapy for T2Ml or myocardial injury. Considering eligibility for randomisation in a clinical trial, each drug was classified according to indications and contraindications for therapy and survival for at least 24 hours following admission. Eligibility criteria for prescription were evaluated against the Summary of Medical Product Characteristics. Consecutive hospital admissions were screened to identify patients with $\geq 1$ high-sensitivity troponin-I value $>99$ th percentile. Endotypes of myocardial injury were adjudicated according to the Fourth Universal Definition of MI. Patients' characteristics and medication were prospectively evaluated.

Results During 1 March to 15 April 2020, 390 patients had a troponin I>URL. Reasons for exclusion: type $1 \mathrm{Ml}$ $n=115$, indeterminate diagnosis $n=42$, lack of capacity $n=14$, death $<24$ hours $n=7$, duplicates $n=2$. Therefore, 210 patients with T2MI/myocardial injury and $174(82.8 \%)$ who survived to discharge were adjudicated for treatment eligibility. Patients who fulfilled eligibility criteria initially on admission and then at discharge were colchicine 25/210 (11.9\%) and 23/174 (13.2\%); eplerenone 57/210 (27.1\%) and $45 / 174$ (25.9\%); ticagrelor 122/210 (58.1\%) and $98 / 174(56.3 \%)$. Forty-six (21.9\%) and $38(21.8 \%)$ patients were potentially eligible for all three drugs on admission and discharge, respectively.

Conclusion A reasonably high proportion of patients may be considered eligible for repurposing novel medical therapy in secondary prevention trials of type $2 \mathrm{Ml} /$ myocardial injury.

\section{BACKGROUND}

Type 2 myocardial infarction (MI) and nonischaemic myocardial injury confer a worse prognosis than type $1 \mathrm{MI},{ }^{1}$ yet there are no evidence-based medical therapies. Highsensitivity troponin assays are recommended in practice guidelines for use in routine clinical practice, leading to more patients with

\section{Key questions}

What is already known about this subject?

- Clinical trials in patients with type 2 myocardial infarction or myocardial injury are limited by the level of heterogeneity and comorbidities within the target population.

- As a result, little evidence is available to provide evidence-based management to these patients, with current treatment strategies focused on empirical secondary prevention.

What does this study add?

- This hypothetical study provides a pragmatic and contemporary insight into the proportion of consecutively screened patients who would be eligible for treatment with three repurposed agents-colchicine, eplerenone and ticagrelor-chosen for their application in other cardiovascular disorders and varied index of contraindications to therapy.

How might this impact on clinical practice?

- Despite a comorbid and elderly patient population $21.8 \%$ of patients would be eligible for all three potential agents in a secondary prevention or $21.9 \%$ in an acute intervention trial when restricting eligibility to include no absolute or relative contraindication.

- This potential population can be increased substantially by allowing patients with relative contraindications, that is, age greater than 65 years; to be considered eligible.

nonischaemic myocardial injury or type 2 MI being diagnosed. ${ }^{2}$ Despite their high mortality, no secondary prevention trials have been undertaken in type $2 \mathrm{MI}$ and prior post-MI trials have focused on type $1 \mathrm{MI}$.

Pharmacotherapeutic strategies for type 2 MI or myocardial injury could involve one of three approaches (1) repurposing, (2) re-evaluation of current guideline-recommended therapies which have mainly been derived from trials involving patients with type $1 \mathrm{MI}$ and (3) novel therapy. The success or failure of delivering a clinical trial is determined by several factors, notably enrolment rates and the safety of the participants. The proportion 
of patients possessing eligibility criteria in the target population is a key uncertainty for the design and implementation of a clinical trial, that is inevitably associated with some level of uncertainty.

Colchicine inhibits neutrophil microtubule formation with anti-inflammatory effects. Recent trials in coronary heart disease have confirmed that colchicine has antiatherosclerotic effects that reduce the risk of cardiovascular events. These trials mainly included patient with type 1 MI leaving an evidence gap for patients with type 2 MI or myocardial injury. ${ }^{3-7}$ Eplerenone is a selective mineralocorticoid receptor antagonist (MRA). MRAs reduce adverse ventricular remodelling relevant to type $2 \mathrm{MI}$ or myocardial injury and improve prognosis overall in patients with left ventricular dysfunction following acute MI. ${ }^{8}$ One of the few clinical trials to describe patients with type 2 MI was PEGASUS-TIMI- $54 .{ }^{10}$ In this trial, 21 162 patients with recent MI (including 13\% with type-2 MI) were randomised to receive ticagrelor (60 $\mathrm{mg}$ or 90 $\mathrm{mg}$ two times per day) or placebo. Ticagrelor reduced the risk of combined cardiovascular mortality, MI or stroke at 3 years (both doses) compared with placebo. ${ }^{10}$

We, therefore, predefined colchicine, eplerenone and ticagrelor as candidate medicines for evaluation in secondary prevention clinical trials in patients with type 2 MI or myocardial injury. The potential eligibility of affected patients for these medicines is unknown. We studied the characteristics of patients hospitalised with type $2 \mathrm{MI}$ and nonischaemic myocardial injury in order to determine the potential eligibility for one or more of these medicines in the theoretical context of enrolment into a randomised, controlled trial of each medication.

\section{METHODS}

\section{Design and setting}

A longitudinal cohort study was undertaken in a large urban academic medical centre (Queen Elizabeth University Hospital, Glasgow, United Kingdom: catchment population $\mathrm{n}=650000$ ) between 1 March and 15 April 2020. The study protocol and proforma were predefined and Caldicott guardian approval for the use of patientidentifiable data was obtained before starting the project. Routinely collected (usual care) data were gathered by clinicians who were members of the usual care medical team, and ethics approval or explicit patient consent was not required.

\section{Screening strategy to identify the target population}

The study had three sequential stages. The screening and adjudications were led by a team of acute medical physicians (MB, TK, OP and RS) supervised by two experienced cardiologists (KM and $\mathrm{CB}$ ). The first step was to identify hospitalised patients with a troponin I $>$ URL based on a screen in realtime of laboratory records. The second stage was to assess the available clinical information for the episode of care in order to determine the endotype according to the fourth Universal Definition of MI (UDMI). The third step focused on the subgroup with a diagnosis of type 2 MI or nonischaemic myocardial injury.

Patients with an elevated troponin-I concentration (Abbott Architect TnI assay) based on a clinically indicated test were identified from laboratory-sourced records of $\geq 1 \mathrm{hs}-T n \mathrm{I}$ result $>99$ th percentile sex-specific upper reference limit (sex-specific URL; 99th centile: men:>34 ng/L; women:>16 $\mathrm{ng} / \mathrm{L}$ ). The acute medical physicians adjudicated individual patient records and assigned endotypes of myocardial injury according to the fourth UDMI in real-time. The adjudications were supported by the cardiologists (KM and $\mathrm{CB}$ ). In cases of diagnostic ambiguity, endotypes were determined by consensus agreement. Only patients with type $2 \mathrm{MI}$ or nonischaemic myocardial injury, subclassified by inciting aetiology (cardiovascular, noncardiovascular), were included in the assessment for drug eligibility.

Electronic patient records (NHS Greater Glasgow and Clyde Health Board: Clinical Portal, Trakcare) were reviewed and details on demographics and medical history were recorded. In-hospital outcomes including mortality and duration of stay were also obtained.

\section{Determining the study population (type $\mathbf{2} \mathbf{M I}$ or myocardial injury)}

Inclusion: (1) index admission with $\geq 1$ hs-TnI value $>99$ th sex-specific URL, (2) $\geq 18$ years or older. Exclusion: (1) indeterminate diagnosis due to incomplete (or inaccessible) electronic patient records. Additional exclusion criteria were assessed prospectively using information that became available during the in-patient stay, (2) deaths $<24$ hours following admission and (3) lack of capacity (dementia/ cognitive impairment).

Endotypes of nonthrombotic myocardial injury (type 2 MI, cardiac nonischaemic myocardial injury, noncardiac nonischaemic myocardial injury) were included in the screening criteria. Those with type $1 \mathrm{MI}$ and other phenotypes of myocardial injury according to the Fourth UDMI were omitted.

\section{Assessment of potential eligibility for novel medical therapy}

We assessed the Electronic Medicines Compendium for the Summary of Medical Product Characteristics (SmPC) for colchicine, eplerenone and ticagrelor. ${ }^{11-13}$ Drug-specific prescribing information were also checked using reference prescribing guidelines ${ }^{414}$ and information from a recent clinical trial of colchicine. ${ }^{15}$

The clinical team assessed each patient according to predefined eligibility criteria for prescription of each drug in the theoretical context of a clinical trial. Eligibility included the absence of (1) a clinical indication for therapy, (2) an absolute contraindication and (3) a relative contraindication (caution). In addition, we took advantage of our recent experience in the COLCOT trial, which randomised patients within 30 days of type $1 \mathrm{MI}$ to colchicine $0.5 \mathrm{mg}$ daily or placebo. In addition to applying the SmPC criteria for colchicine, we also included the criteria for the COLCOT trial as a framework to inform the eligibility criteria and inclusion in 
a type 2 MI or myocardial injury trial. ${ }^{15}$ The flow diagram for our study is shown in figure 1 .

\section{Timing of the eligibility assessment in relation to the clinical care pathway}

Clinical status may evolve during an episode of care, and this influences the status of drug prescription. Accordingly, we predefined a sequential approach to determining eligibility for the study medicines. The clinical criteria for each medicine were sequentially assessed at two study time points: (1) following admission ( $\leq 24$ hours) and (2) at discharge. The rationale for this approach has recently been supported by evidence that the benefit of colchicine in patients with recent MI may be greatest when initiated $<3$ days of the index MI. ${ }^{15}$

\section{COVID-19}

This study was undertaken during the SARS-CoV2 (COVID19) pandemic. COVID-19-positive patients were coded based on either laboratory evidence of SARS-CoV-2 infection by real-time PCR (Roche Cobas 6800 or Seegene Allplex
2019-nCoV) assay and/or positive radiological diagnosis (chest CT, chest radiograph) but negative biospecimen. The pandemic may have enriched the study population with patients diagnosed with type 2 MI or myocardial injury.

\section{Statistics}

The statistical analyses were performed using IBM Statistics SPSS (V. 24.0). $\chi^{2}$ (or Fisher's exact test) are calculated for categorical characteristic variables. Kruskal-Wallis one-way analysis of variance tests were performed for multiple independent numerical continuous variables.

\section{RESULTS}

Three hundred and ninety patients were acutely hospitalised and had an elevated hs-TnI between 1 March and 15 April 2020. Of these, 42 had an indeterminate diagnosis due to inaccessible electronic records and two records were excluded due to duplication. A further 115 patients with type $1 \mathrm{MI}$ were then excluded from the drug eligibility assessment.

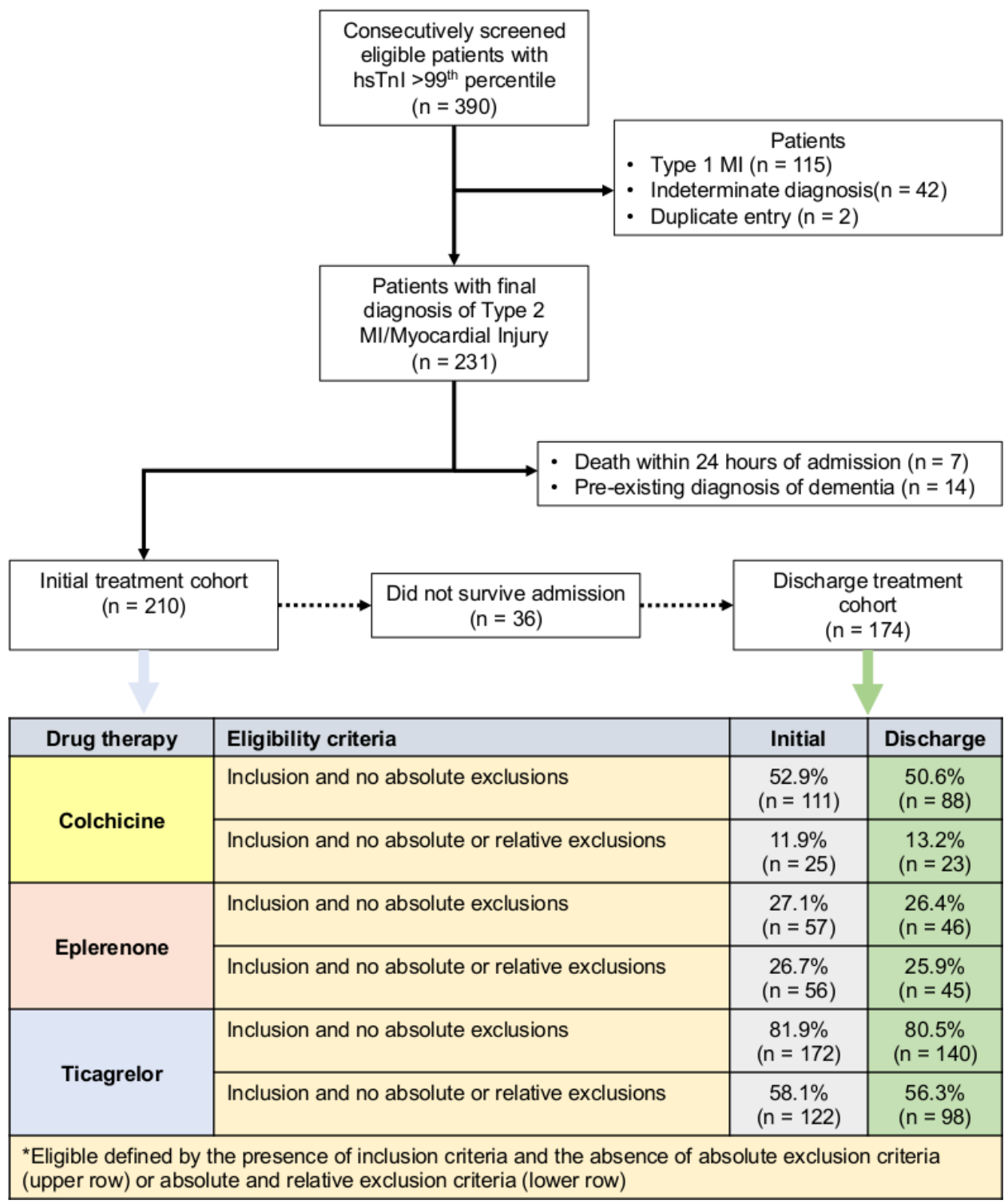

Figure 1 Flow diagram and summary of patient eligibility. MI, myocardial infarction. 


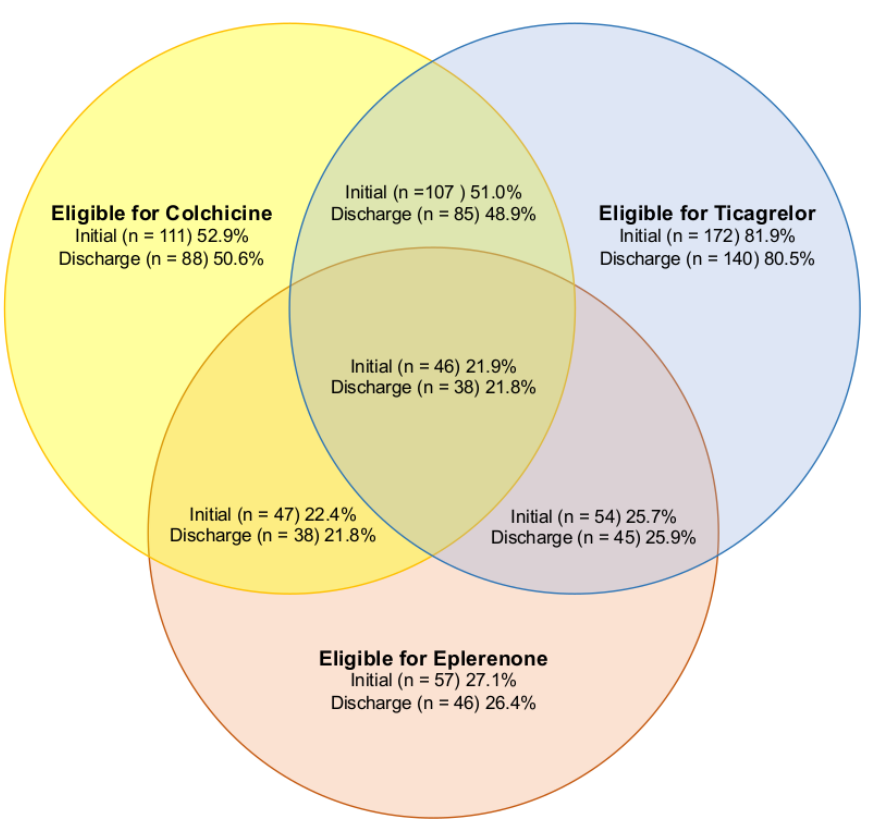

Figure 2 Flow diagram and summary of eligibility for novel medical therapy with no absolute contraindication for therapy.

Of the remaining 231 patients eligible for drug eligibility assessment, an index diagnosis of type 2 MI occurred in 79 patients and a diagnosis of nonischaemic myocardial injury occurred in 152 patients (cardiac $n=69$; noncardiac $n=83$ ).

\section{Eligibility analysis}

Figures 1 and 2 illustrate patient flow from screening laboratory troponin results, endotype evaluation and adjudication of drug-specific eligibilities. Of 231 patients with type $2 \mathrm{MI}$ or nonischaemic myocardial injury who might be considered as potential candidates for participation in a clinical trial, 210 patients $(49.0 \%$ women $)$ were considered for inclusion following an initial assessment and 21 were excluded (death within 24 hours of admission: $\mathrm{n}=7$ (3.0\%); lack of capacity to consent (dementia/ cognitive impairment): $\mathrm{n}=14(6.1 \%))$. Of these, at the point of discharge, 174 patients $(51.7 \%$ women) were eligible, with exclusion of 57/231 (in-hospital death: $\mathrm{n}=43(18.6 \%)$; lack of capacity to consent (dementia/ cognitive impairment): $\mathrm{n}=14(6.1 \%))$.

\section{Patient characteristics across groups}

Online supplemental table 1 summarises the participants' characteristics. Age, sex and ethnicity were not different between the endotype groups. Compared with the excluded type $1 \mathrm{MI}$ group, patients with type $2 \mathrm{MI}$ or nonischaemic myocardial injury had higher 10-year predicted mortality, but quantitatively smaller initial and peak hsTnI values. Hospital readmission rate was comparable between all endotypes with one in five patients readmitted (median, 19.0 days (IQR, 25.0)). Invasive coronary angiography was infrequently performed in patients with type $2 \mathrm{MI}(1.3 \%, \mathrm{n}=1)$, noncardiac myocardial injury $(1.2 \%, \mathrm{n}=1)$ and cardiac myocardial injury $(5.8 \%, \mathrm{n}=4)$. Percutaneous coronary intervention was undertaken in one patient with cardiac myocardial injury and no patients in any of these groups underwent surgical revascularisation.

Infections (COVID-19 and non-COVID-19) were more frequent in patients with type 2 MI or nonischaemic myocardial injury in addition to anaemia, structural heart disease and tachyarrhythmia.

\section{Prescribed medications during standard clinical care}

The prescriptions of cardiovascular medicines at the point of hospitalisation and at the point-of-discharge are summarised in online supplemental table 2. There were no differences in cardiovascular drug prescriptions by endotype. Four patients were prescribed colchicine therapy in-hospital/at discharge.

\section{Initial treatment eligibility group}

Relative and absolute contraindications were evaluated. Overall, 179 (85.2\%) of the 210 potentially eligible patients met the essential defined criteria for colchicine. One hundred and eleven (52.9\% of initial cohort) met essential inclusion criteria and had no absolute contraindication to therapy (online supplemental table 3). Fewer patients $(40.0 \%$; $=84$ ) were eligible once study exclusions (in-hospital death within 24 hours, ineligible by incapacity) were applied; fewer still $(11.9 \% ; n=25)$ were eligible after considering all of the relative, absolute and study-defined exclusions. Therefore, $25 / 210(11.9 \%)$ of the initial cohort were eligible for inclusion in a clinical trial of colchicine, if informed consent was given.

Eplerenone could be initiated during admission in $57 / 210(27.1 \%)$ and $56(26.7 \%)$ patients without absolute or combined absolute/cautionary contraindications, respectively (see online supplemental table 4). A complete breakdown of the criteria and grading are provided in online supplemental tables 3 to 5 .

Finally, ticagrelor could potentially be initiated as an acute intervention in $172(81.9 \%)$ of 210 patients who fulfilled the inclusion criteria and lacked an absolute contraindication. When both absolute and relative (cautionary) contraindications were applied, $122(58.1 \%)$ remained theoretically eligible for treatment with ticagrelor (see online supplemental table 5).

\section{Discharge treatment eligibility group}

Thirty-six (17.1\%) of 210 patients died during the index admission; including 22 who died from COVID-19. One hundred and seventy-four (82.9\%) of 210 patients were included in the subgroup for whom data were available at discharge. Of these, 145 (83.3\%) patients met the essential criteria for inclusion.

Following adjustment for contraindications, cautioned use or patient ineligibility for study reasons, 23 of 174 patients were theoretically eligible for treatment with colchicine at discharge. The percentage of patients potentially eligible for colchicine at the point of discharge increased to $44.8 \%$ if stringency was to be relaxed and cautions for prescribing (relative contraindications) were discounted: elderly age 
( $\mathrm{n}=113)$, concomitant systemic steroids $(\mathrm{n}=11)$, active or recent pneumonia $(n=20)$ or left ventricular ejection fraction (LVEF) less than $35 \%(\mathrm{n}=20)$.

Eplerenone could potentially be commenced in 45 out of $174(25.9 \%)$ patients eligible for trial inclusion at discharge.

Five patients had an absolute contraindication to ticagrelor with 140 of $174(80.5 \%)$ remaining eligible for treatment at discharge. With additional consideration for cautionary factors applied, then the proportion of patients potentially eligible for receiving ticagrelor reduced to 98/145 (56.3\%) (online supplemental table 5).

\section{Eligibility for all three medications}

Considering eligibility for all three medicines, 46 (21.9\%) and $38(21.8 \%)$ patients were eligible for all three medications on admission and at discharge, respectively (figure 2).

\section{DISCUSSION}

We have prospectively documented the potential eligibility of patients with type 2 MI or nonischaemic myocardial injury for repurposing three candidate medicines in the theoretical context of a randomised, controlled trial. The data are contemporary, prospectively evaluated and relatively unselected. Our results should help to derisk the design and implementation of a future clinical trial in type $2 \mathrm{MI}$ or nonischaemic myocardial injury involving one of these medicines.

We considered the potential eligibility of the patients for these candidate therapies in relation to the acute care pathway, first following hospital admission and second at discharge. We found that $111(52.9 \%)$ patients admitted with type $2 \mathrm{MI}$ or nonischaemic myocardial injury might be eligible without absolute contraindications for a trial of early (in-hospital) initiation of colchicine, 57 (27.1\%) would be eligible for secondary prevention trials with eplerenone and 172 (81.9\%) with ticagrelor. Considering patients surviving through to discharge, 88 (50.6\%), 140 $(80.5 \%)$ and $46(26.4 \%)$ were eligible for colchicine, eplerenone and ticagrelor therapy, respectively. Age $>65$ years was a relative caution for the prescription of colchicine and eplerenone.

Repurposing an established therapy is, theoretically, an attractive proposition since the safety of the medicine is generally well understood, derisking the trial. Considering efficacy, supporting information on the efficacy of repurposing candidates may already exist in a related disease area. Patients with type 2 MI or nonischaemic myocardial injury have distinct characteristics, including older age and prevalent comorbidity. Furthermore, enrolling patients into a drug trial during acute care raises presents particular challenges to trial recruitment, including the feasibility of obtaining written informed consent, meeting enrolment milestones and, of course, the type of clinical endpoints to assess the safety and efficacy of the repurposed medicine. A White Paper review of type 2 MI by DeFilippis $e a^{16}$ identifies a paucity of clinical evidence from randomised, controlled, secondary prevention. In our study, we attempted to enhance the relevance of the results for colchicine by drawing on relevant criteria from the COLCOT trial, which reflects contemporary prescribing, although in patients with type $1 \mathrm{MI}$.

\section{Colchicine}

Colchicine is an anti-inflammatory drug extracted from Colchicum autumnale (autumn crocus). Colchicine is guideline indicated in the management of myopericarditis and, recently, has been shown to be an effective treatment in reducing composite cardiovascular end points including stroke and readmission with angina in patients' postacute MI and in stable coronary artery disease ${ }^{3-517}$ Colchicine is a tricyclic, lipid-soluble alkaloid reaching peak plasma volume $60 \mathrm{~min}$ after oral administration with long half-life duration. Increased concentrations within neutrophils are in keeping with its potent anti-inflammatory properties. ${ }^{18}$ While colchicine has been used therapeutically for thousands of years, it was not approved by the FDA until 2009. Colchicine is now indicated therapy for pericarditis and gout, and it may lower the incidence of post-operative or post-ablation atrial fibrillation. ${ }^{6} 71920$ In patients with either acute MI or stable coronary disease colchicine reduces the need for repeat revascularisation, which may be explained by its antiatherosclerotic effects ${ }^{4521-23}$ Colchicine has been investigated in large randomised controlled trials in patients with COVID-19. Preprint data from the COLCORONA trial described a reduction in the composite of hospitalisation and mortality among nonhospitalised patients with COVID-19 when treated with colchicine versus placebo ( $4.6 \%$ vs $6.0 \%$; OR, $0.75 ; 95 \%$ CI 0.57 to 0.99; $\mathrm{p}=0.04) .{ }^{24}$ In hospitalised patients with COVID-19, the RECOVERY investigators found no effect of colchicine on 28-day mortality (20\% colchicine vs $19 \%$ usual care alone; risk ratio 1.02 (95\% CI 0.94-1.11); $\mathrm{p}=0.63) .^{25}$ However, it is unclear whether colchicine might be beneficial in patients with myocardial injury or type $2 \mathrm{MI}$ and COVID-19 disease as data in this subgroup are lacking.

The most common side effect of oral colchicine administration is gastrointestinal upset, occurring in up to $20 \%$ of patients and $20 \%$ of reasons for discontinuation. Less common (combined $<5 \%$ ) potential side effects include myalgia, rash, alopecia or hepatotoxicity. Pre-existing liver disease or poor creatinine clearance increase the likelihood of side effects. In the Australian COPS Trial and LoDoCo2 Trials, colchicine was associated with an increase in noncardiovascular deaths. ${ }^{517}$ Colchicine has immunosuppressive effects and in the COLCOT trial, pneumonia was more common in colchicine-treated patients $(\mathrm{p}=0.03) .{ }^{4}{ }^{26}$ A systematic review and metaanalysis of 35 randomised control trials have not borne out an increased risk of infection with colchicine. ${ }^{27}$

\section{Eplerenone}

Aldosterone plays an important role in the pathophysiological mechanisms of heart failure and mediates the 
deleterious downstream effects of renin-angiotensinaldosterone system activation, including endothelial dysfunction, cardiovascular inflammation, myocardial fibrosis, ventricular remodelling and increased arrhythmogenicity. Plasma concentrations early post-MI are independently associated with increased all-cause mortality. ${ }^{28}$ ${ }_{29}$ MRA (nonselective: spironolactone; selective: eplerenone) reduce both the risk of death and hospitalisation in selected patients following acute MI with LVEF $\leq 40 \%$ and clinically evident heart failure. ${ }^{9} 290$ REMINDER was a randomised, placebo-controlled, double-blind trial of eplerenone in patients presenting with acute MI without heart failure. ${ }^{31}$ After 10.5 months, the primary endpoint occurred in $92(18.2 \%)$ and 149 patients $(29.4 \%)$ in the eplerenone and placebo groups, respectively (HR 0.58 ; $95 \%$ CI 0.45 to $0.76 ; \mathrm{p}<0.01)$. This result was driven by a treatment-related reduction in NTproBNP. In the HOMAGE trial, patients with risk factors for heart failure (mean age 73 years, $26 \%$ women, $71 \%$ prior $\mathrm{MI}$ ), including an increased NT-proBNP and no prior history of heart failure, were randomised to receive spironolactone or standard care. ${ }^{32}$ In addition to a treatment-related reduction in NT-proBNP (mean difference $-57 ; 95 \%$ CI -81 to $-33 \mathrm{ng} / \mathrm{L} ; \mathrm{p}<0.0001)$ spironolactone also reduced type 1 collagen degradation reflected by a reduction in carboxy terminal propeptide of type 1 procollagen (mean difference $-8.1 ; 95 \%$ CI -11.9 to $-4.3 \mu \mathrm{g} / \mathrm{L} ; \mathrm{p}<0.0001$ ) (primary endpoint). Considering mechanisms, this antifibrotic effect may reduce left ventricular stiffness, which would lead to a favourable reduction in NT-proBNP. Potentially, MRA therapy may be beneficial to which would lead to a favourable reduction in NT-proBNP. Potentially, MRA therapy may be beneficial to patients with type 2 MI and this possibility merits prospective evaluation.

Spironolactone has antiandrogenic side effects such as gynecomastia or impotence. The development of selective nonsteroidal MRAs such as eplerenone, and MR modulators such as finerenone, which reduce the likelihood of hyperkalaemia, have a more favourable side effect profile. ${ }^{30}$

\section{Ticagrelor}

A reversible cyclopentyl triazolopyrimidine, orally active, selective adenosine diphosphate $\left(\mathrm{P}_{2} \mathrm{Y}_{12}\right)$ receptor antagonist-ticagrelor is indicated for patients with acute coronary syndromes. ${ }^{33}$ The platelet inhibition and patient outcome trial found that ticagrelor reduced composite MI, stroke or death compared with clopidogrel in patients who were both medically managed or who underwent revascularisation. ${ }^{34}$ However, ticagrelor increased major bleeding compared with placebo and aspirin. ${ }^{35}$ Patients with type 2 MI or myocardial injury may have an increased risk of 30-day major adverse cardiovascular events compared with type 1 MI (20\% vs $9 \%)$, highlighting competing risks and benefits.

\section{Additional therapies}

We have selected novel therapies based on their established efficacy and safety in other forms of cardiovascular disease. There are limited data available on other candidate therapies in type 2 MI, including beta blockers, statins and angiotensin-converting enzyme receptor inhibitors/angiotensin receptor blockers and proprotein convertase subtilisin/kexin type 9 (PCSK9) inhibitors. ${ }^{3637}$ Favourable effects of alirocumab, when added to intensive statin therapy, were reported in a prespecified analysis from the ODYSSEY OUTCOMES trial (effects of alirocumab on types of MI) ${ }^{38}$ The novel findings were a reduction in the incidence of recurrent MI, either type 1 or type 2, in patients with elevated low-density lipoprotein cholesterol after an index acute coronary syndrome. Alirocumab was well tolerated with a favourable sideeffect profile. The findings in prior studies, and our own, support the rationale for randomised, controlled, clinical trials.

\section{Limitations}

While patients have been designated as potentially eligible within the index admission, the diagnosis of type 2 MI or myocardial injury may not be suspected or confirmed based on the initial troponin I measurement. In our population, $20.5 \%$ had changes in diagnosis arising between the initial aetiology of hsTnI elevation and the final diagnosis of type $2 \mathrm{MI}$, including 32 (15.2\%) initially coded as type $1 \mathrm{MI}$. The relatively small number of patients with type 2 MI or myocardial injury identified within the study period is a limitation.

COVID-19 was a primary or secondary diagnosis in 35 $(16.7 \%)$ of the initially eligible patients and a significant primary cause of in-hospital mortality for 22 patients (10.4\%). COVID-19 and its sequalae are likely to be a relevant public health problem for the foreseeable future. ${ }^{39}$ COVID-19 is associated with myocardial injury and type 2 MI in unscheduled care. ${ }^{40}$

\section{CONCLUSIONS}

Patients with type $2 \mathrm{MI}$ or myocardial injury are commonly multimorbid. Despite this, a reasonably high proportion of these patients may be considered eligible for repurposing novel medical therapy in secondary prevention trials.

Twitter Robert Sykes @_rsykes, Kenneth Mangion @kenneth_mangion and Colin Berry@ColinBerryMD

Contributors The manuscript was drafted by RS and reviewed by MB, KM and CB. $\mathrm{CB}$ designed and instigated the project. All authors contributed to the collection or adjudication of data.

Funding Professor Colin Berry is supported by research funding from the British Heart Foundation (PG/17/2532884; RE/13/5/30177; RE/18/6/34217) and Medical Research Council (UKRI/MRC MR/S018905/1).

Competing interests Professor Colin Berry is employed by the University of Glasgow which holds consultancy and research agreements for his work with companies that have commercial interests in the diagnosis and treatment of angina. The companies include Abbott Vascular, Astra Zeneca, Boehringer Ingelheim, GSK, HeartFlow, Menarini, Novartis, and Siemens Healthcare. The other authors do not have any potential conflicts of interest.

Patient consent for publication Not required.

Provenance and peer review Not commissioned; externally peer reviewed. 
Data availability statement The data that support the findings of this study are available on request from the corresponding author. The data are not publicly available due to privacy or ethical restrictions.

Open access This is an open access article distributed in accordance with the Creative Commons Attribution Non Commercial (CC BY-NC 4.0) license, which permits others to distribute, remix, adapt, build upon this work non-commercially, and license their derivative works on different terms, provided the original work is properly cited, appropriate credit is given, any changes made indicated, and the use is non-commercial. See: http://creativecommons.org/licenses/by-nc/4.0/.

ORCID iD

Robert Sykes http://orcid.org/0000-0003-1010-5474

\section{REFERENCES}

1 Briscoe M, Sykes R, Krystofiak T. Clinical significance of troponin elevation in a contemporary hospitalized population: Endotypes, morbidity and in-hospital mortality. AHA Scientific Sessions 2020.

2 Collet J, Thiele H, Barbato E. Corrigendum to: 2020 ESC guidelines for the management of acute coronary syndromes in patients presenting without persistent ST-segment elevation: the task force for the management of acute coronary syndromes in patients presenting without persistent ST-segment elevation of the European Society of cardiology (ESC). Eur Heart J 2021:ehaa575.

3 Adler Y, Charron P, Imazio M, et al. "2015 ESC Guidelines for the diagnosis and management of pericardial diseases: The task force for the Diagnosis and Management of Pericardial Diseases of the European Society of Cardiology (ESC),". Eur Heart J 2015;36:2921-64.

4 Tardif J-C, Kouz S, Waters DD, et al. Efficacy and safety of low-dose colchicine after myocardial infarction. N Engl J Med 2019;381:2497-505.

5 Nidorf SM, Fiolet ATL, Mosterd A, et al. Colchicine in patients with chronic coronary disease. N Engl J Med 2020;383:1838-47.

6 Imazio M, Bobbio M, Cecchi E, et al. Colchicine in addition to conventional therapy for acute pericarditis: results of the colchicine for acute pericarditis (cope) trial. Circulation 2005;112:2012-6.

7 Deftereos S, Giannopoulos G, Kossyvakis C, et al. Colchicine for prevention of early atrial fibrillation recurrence after pulmonary vein isolation: a randomized controlled study. J Am Coll Cardiol 2012;60:1790-6.

8 Hayashi M, Tsutamoto T, Wada A, et al. Immediate administration of mineralocorticoid receptor antagonist spironolactone prevents postinfarct left ventricular remodeling associated with suppression of a marker of myocardial collagen synthesis in patients with first anterior acute myocardial infarction. Circulation 2003;107:2559-65.

9 Pitt B, Remme W, Zannad F, et al. Eplerenone, a Selective Aldosterone Blocker, in Patients with Left Ventricular Dysfunction after Myocardial Infarction," NEJM 2003;348:1309-21.

10 Bonaca MP, Wiviott SD, Morrow DA, et al. Reduction in subtypes and sizes of myocardial infarction with ticagrelor in PEGASUS-TIMI 54. J Am Heart Assoc 2018:7:1-9.

11 Wockhardt UK Ltd. The electronic medicines compendium (emc), "Colchicine 500 micrograms Tablets,", 2019. Available: https://www. medicines.org.uk/emc/product/6415/smpc\# [Accessed 11 October 2020].

12 Consilient Health Ltd. The electronic medicines compendium (emc), "Eplerenone 25 mg film-coated tablets,", 2018. Available: https:// www.medicines.org.uk/emc/product/1981/smpc [Accessed 11 October 2020].

13 AstraZeneca UK Limited. The electronic medicines compendium (emc), "Brilique $90 \mathrm{mg}$ film coated tablets,", 2019. Available: https:// www.medicines.org.uk/emc/product/5767/smpc [Accessed 11 October 2020]

14 Joint Formulary Committee. "British National Formulary (online),", 2020. Available: http://www.medicinescomplete.com [Accessed September 2020].

15 Bouabdallaoui N, Tardif J-C, Waters DD, et al. Time-to-treatment initiation of colchicine and cardiovascular outcomes after myocardial infarction in the colchicine cardiovascular outcomes trial (COLCOT). Eur Heart J 2020;41:4092-9.

16 DeFilippis AP, Chapman AR, Mills NL, et al. Assessment and treatment of patients with type 2 myocardial infarction and acute nonischemic myocardial injury. Circulation 2019;140:1661-78.

17 Tong DC, Quinn S, Nasis A, et al. Colchicine in patients with acute coronary syndrome: the Australian COPS randomized clinical trial. Circulation 2020;142:1890-1900.
18 Yan BP, Tan G-M. What's Old is New Again - A Review of the Current Evidence of Colchicine in Cardiovascular Medicine. Curr Cardiol Rev 2017;13:130-8.

19 Imazio M, Brucato A, Cemin R, et al. Colchicine for recurrent pericarditis (CORP): a randomized trial. Ann Intern Med 2011;155:409-14.

20 Imazio M, Brucato A, Ferrazzi P, et al. Colchicine for prevention of postpericardiotomy syndrome and postoperative atrial fibrillation: the COPPS-2 randomized clinical trial. JAMA 2014;312:1016-23.

21 Deftereos S, Giannopoulos G, Raisakis K, et al. Colchicine treatment for the prevention of bare-metal stent restenosis in diabetic patients. J Am Coll Cardiol 2013:61:1679-85.

22 Nidorf M, Thompson PL. Effect of colchicine ( $0.5 \mathrm{mg}$ twice daily) on high-sensitivity C-reactive protein independent of aspirin and atorvastatin in patients with stable coronary artery disease. $\mathrm{Am} \mathrm{J}$ Cardiol 2007;99:805-7.

23 Nidorf SM, Eikelboom JW, Budgeon CA, et al. Low-dose colchicine for secondary prevention of cardiovascular disease. J Am Coll Cardiol 2013;61:404-10.

24 Tardif J, Bouabdallaoui N, L'Allier, D PL. "Efficacy of Colchicine in Non-Hospitalized Patients with COVID-19,". medRxiv 2021.

25 T. R. T. C. Investigators. "RECOVERY trial closes recruitment to colchicine treatment for patients hospitalised with COVID-19," Nuffield department of Population Health, 2021. Available: https:// www.recoverytrial.net/news/recovery-trial-closes-recruitmentto-colchicine-treatment-for-patients-hospitalised-with-covid-19 [Accessed 11 April 2021].

26 Tsai T-L, Wei JC-C, Wu Y-T, et al. The association between usage of colchicine and pneumonia: a nationwide, population-based cohort study. Front Pharmacol 2019;10:908.

27 Stewart S, Yang KCK, Atkins K, et al. Adverse events during oral colchicine use: a systematic review and meta-analysis of randomised controlled trials. Arthritis Res Ther 2020;22:28.

28 Beygui F, Collet J-P, Benoliel J-J, et al. High plasma aldosterone levels on admission are associated with death in patients presenting with acute ST-elevation myocardial infarction. Circulation 2006;114:2604-10.

29 De Mello WC. Beneficial effect of eplerenone on cardiac remodelling and electrical properties of the failing heart. $J$ Renin Angiotensin Aldosterone Syst 2006;7:40-6.

30 Berbenetz NM, Mrkobrada M. Mineralocorticoid receptor antagonists for heart failure: systematic review and meta-analysis. BMC Cardiovasc Disord 2016;16:246.

31 Montalescot G, Pitt B, Lopez de Sa E, et al. Early eplerenone treatment in patients with acute ST-elevation myocardial infarction without heart failure: the randomized double-blind reminder study. Eur Heart J 2014:35:2295-302.

32 Cleland JGF, Ferreira JP, Mariottoni B, et al. The effect of spironolactone on cardiovascular function and markers of fibrosis in people at increased risk of developing heart failure: the heart 'OMics' in AGEing (HOMAGE) randomized clinical trial. Eur Heart $J$ 2021;42:684-96.

33 Dhillon S. Ticagrelor: a review of its use in adults with acute coronary syndromes. Am J Cardiovasc Drugs 2015;15:51-68.

34 James S, Åkerblom A, Cannon CP, et al. Comparison of ticagrelor, the first reversible oral P2Y12 receptor antagonist, with clopidogrel in patients with acute coronary syndromes: rationale, design, and baseline characteristics of the PLATelet inhibition and patient Outcomes (PLATO) trial. Am Heart J 2009;157:599-605.

35 Steg PG, Bhatt DL, Simon T, et al. Ticagrelor in patients with stable coronary disease and diabetes. N Engl J Med 2019;381:1309-20.

36 Lindahl B, Baron T, Erlinge D, et al. Medical therapy for secondary prevention and long-term outcome in patients with myocardial infarction with nonobstructive coronary artery disease. Circulation 2017;135:1481-9.

37 Sandoval Y, Smith SW, Sexter A, et al. Type 1 and 2 myocardial infarction and myocardial injury: clinical transition to high-sensitivity cardiac troponin I. Am J Med 2017;130:1431-9.

38 Schwartz GG, Steg PG, Szarek M, et al. Alirocumab and cardiovascular outcomes after acute coronary syndrome. $N$ Engl J Med 2018;379:2097-107.

39 Anderson RM, Hollingsworth TD, Baggaley RF, et al. COVID-19 spread in the UK: the end of the beginning? Lancet 2020;396:587-90.

40 Briscoe M, Sykes R, Krystofiak T, et al. Clinical significance of coronavirus disease 2019 in hospitalized patients with myocardial injury. Clin Cardiol 2021;44:332-9. 